\title{
Coupled slow and delta oscillations between cuneothalamic and thalamocortical neurons in the chloralose anesthetized cat
}

\author{
Jorge Mariño, Luis Martinez, Antonio Canedo* \\ Department of Physiology, Faculty of Medicine, Santiago de Compostela, Spain
}

Received 23 September 1996; revised version received 16 October 1996; accepted 16 October 1996

\begin{abstract}
Simultaneous recordings were obtained from cuneothalamic (extracellular) and thalamocortical (intracellular) cells in chloralose anesthetized cats. It was found that cuneothalamic neurons present slow rhythmicity $(0.1-1 \mathrm{~Hz})$ tightly coupled to slow oscillations of thalamocortical neurons. This coupling was not due to a direct synaptic linkage but rather produced by other (s) structure (s) probably the cortex. Furthermore, the cuneothalamic neurons also showed delta rhythms (1-4 Hz) coherently oscillating with the delta rhythms of thalamocortical cells which suggests that these rhythms are more widespread than previously thought, and may be a general phenomenon characterizing quiet sleep in multiple structures.
\end{abstract}

Keywords: Cuneate nucleus; Ventroposterolateral thalamic nucleus; Simultaneous recording; Cat

The cuneate nucleus $(\mathrm{CN})$ receives and processes information from the forelimbs. The middle of the nucleus $(0-4$ $\mathrm{mm}$ caudal to the obex) is divided in a core region that predominantly receives cutaneous input and where the cuneothalamic cells predominate; and a shell [6]. This middle nuclear region corresponds with the clusters zone first described by Kuypers and Tuerk [7]. The shell consists of a ventral region in which deep input prevails, and a series of neurons in the medial, lateral and dorsal borders of the cuneate that receive a combination of cutaneous and deep input. The great majority of the cells in the clusters zone project almost exclusively to the nucleus ventralis posterolateralis (VPL) of the contralateral thalamus.

Steriade et al. [14-16] described a slow oscillation $(<1$ $\mathrm{Hz}$ ) grouping the other two sleep rhythms (spindle, 7-14 $\mathrm{Hz}$ and delta, 1-4 $\mathrm{Hz}$ oscillations) in slowly recurring waves. This slow oscillation is generated by intracortical networks including pyramidal-shaped neurons and interneurons. It is transferred to the reticularis thalami nucleus and, after a delay of 100-200 ms, to thalamocortical cells. Steriade et al. [18] predicted that all major targets of neocortical neurons might display the slow rhythm. According

\footnotetext{
* Corresponding author. Tel.: +34 81 582658, ext. 2292; fax: +34 81 574145; email: fsancala@usc.es
}

to this prediction, the cuneate nucleus might also display the slow rhythm since it receives abundant corticofugal innervation $[1,2,10,12]$. Therefore, we directed our attention to this possibility and recorded simultaneously in the cuneate (extracellular) and in the contralateral VPL nucleus (intracellular) of the anesthetized cat.

A total of eight cats of either sex $(2.5-3.5 \mathrm{~kg})$ were anesthetized ( $\alpha$-chloralose, $60 \mathrm{mg} / \mathrm{kg}$, i.p.) and artificially respired. After exposing the dorsal medulla, tungsten recording electrodes were inserted within the middle main cuneate nucleus from the level of the obex to 4 $\mathrm{mm}$ caudal to it. A bilateral pneumotorax was routinely carried out to minimize respiratory and pulsatile movements. A bipolar stimulating electrode was positioned in the contralateral medial lemniscus (ML) at A2, to identify antidromically cuneothalamic cells according to standard criteria $[4,9,10]$. A set of three bipolar stimulating electrodes were inserted in the internal capsule (IC; ipsilateral to the ML electrode at A18) to identify antidromically VPLthalamocortical neurons. A craniotomy was performed over the ventrobasal thalamus and micropipettes filled with a solution buffered to $\mathrm{pH} 7.2$ containing (in $\mathrm{mM}$ ): $\mathrm{KCl}, 20 ; \mathrm{K}^{+}$-acetate, 90; $\mathrm{MgCl}_{2}, 3 ; \mathrm{CaCl}_{2}, 1$; HEPES, 40; EGTA, 3; GTP, 0.4; and $\mathrm{Na}_{2} \mathrm{ATP}$, 4, were lowered into the VPL using a motorized microdrive. The solution 
was adjusted to 300 mosm with saccharose. The resistance of the electrodes measured in vivo ranged from 10 to 20 $\mathrm{M} \Omega$. Further histological procedures demonstrated the correct placement of both stimulating and recording electrodes.

Simultaneous recordings were obtained from cuneothalamic and VPL-thalamocortical neurons. Intracellular current-clamp records of thalamocortical cells revealed their cortically-induced slow rhythms (Fig. 1A, upper record; autocorrelation in Fig. 1C, upper panel) which were tightly coupled to the extracellular activity simultaneously recorded within the cuneate (Fig. 1A, lower record; autocorrelation in Fig. 1C, lower panel). The firing of the cuneothalamic cell coincided in time with the hyperpolarizing period of the thalamocortical neuron (cross-correlation in Fig. 1D). This strong coupling between the activities of cuneothalamic and thalamocortical cells did not depend on a monosynaptic linkage. In effect, when the cuneothalamic spikes were used to compile spike triggered averages of the intracellular synaptic noise of thalamocortical cells, a direct synaptic connection in the sampled pairs $(n=8)$ was not revealed (Fig. 1E). Therefore, the coupling could be imposed by the cortex or, alternatively, reflect intrinsic cuneate oscillations with a constant time window respect to the thalamocortical rhythmicity in the chloralose anesthetized cat. Prethalamic neurons such as those of the cuneate of the cat [3] and those of the deep dentate cerebellar nucleus of both the rat [11] and the guinea pig [8] do possess intrinsic membrane properties allowing them to oscillate at fast frequencies. However, the cuneate slow rhythmicity described in this report is probably due to the effect exerted by corticofugal projections and not to intrinsic properties of cuneate neurons.

The thalamocortical cells have two different modes of operation: an oscillatory and a tonic (transfer) mode. The oscillatory mode is a thalamic intrinsically generated phenomenon since the membrane potential of the thalamocortical cells, at rest, oscillates within the spindle frequency $(7-14 \mathrm{~Hz})$ in absence of cortical and subcortical inputs [5]. The spindles consist of rhythmic long-lasting hyperpolarized periods caused by the activity of the GABAergic reticularis thalami neurons (the spindle pacemakers), followed by postinhibitory rebound bursts [13]. Fig. 1A,B (upper records) show the slow rhythms of a thalamocortical cell. Spindle-like oscillations appeared during the hyperpolarizing periods (Fig. 1B, upper record) (see also Fig. 9 of Ref. [16]). The cuneothalamic neuron simultaneously recorded (Fig. 1B, lower record) also showed extracellular single spike activity within the spindle range that seemed to be time-locked to the thalamic spindle-like rhythmicity. However, autocorrelation (Fig. 1C, inset) and spike-triggered averages (STA) (Fig. 1E) revealed coupled delta-like oscillations in the thalamocortical and cuneothalamic neurons. Thus, the role of the corticothalamic cells in imposing periodic inhibitions over thalamocortical neurons through the reticularis tha-
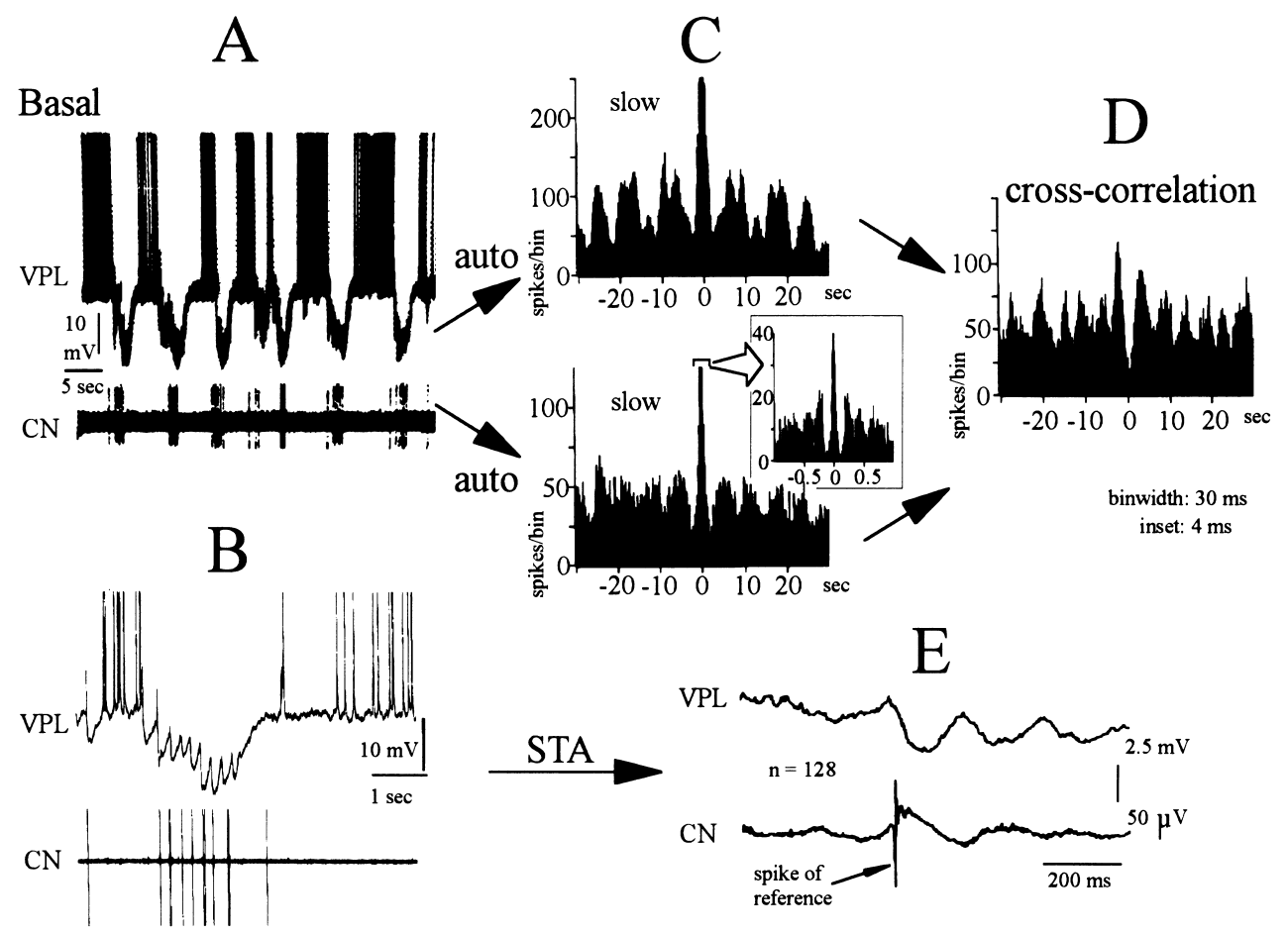

Fig. 1. Slow and delta coupled oscillations between cuneate and thalamic cells. (A,B) Simultaneous records of a ventroposterolateral thalamocortical cell (VPL) and a cuneothalamic (CN) neuron. (C-E) The autocorrelations (C) revealed slow and delta (inset in (C)) oscillations, and cross-correlation revealed a tight coupling between both cells with the rhythmic extracellular cuneate discharge coinciding temporarily with the hyperpolarizing periods of the thalamic cell (D). Spike-triggered averages compiled using the spikes of the cuneate cell as triggers (128 trigger events; the averages of the cuneate cell with respect to itself) demonstrated coherent oscillations in the delta range (E). 


\section{A.- VPL cell}
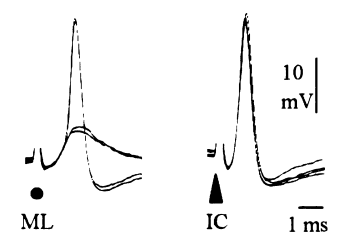

\section{B.- $\mathrm{CN}$ cells}

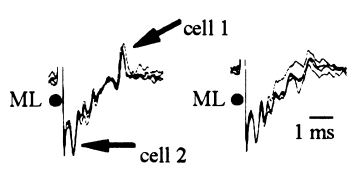

\section{D.- Auto-correlations}
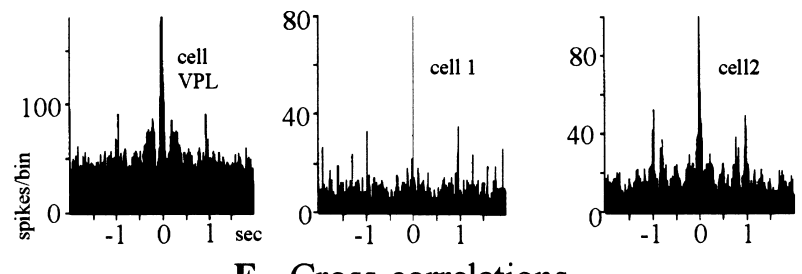

\section{E.- Cross-correlations}
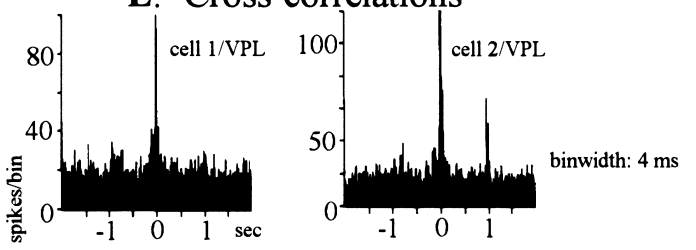

F.- Spike-triggered averages
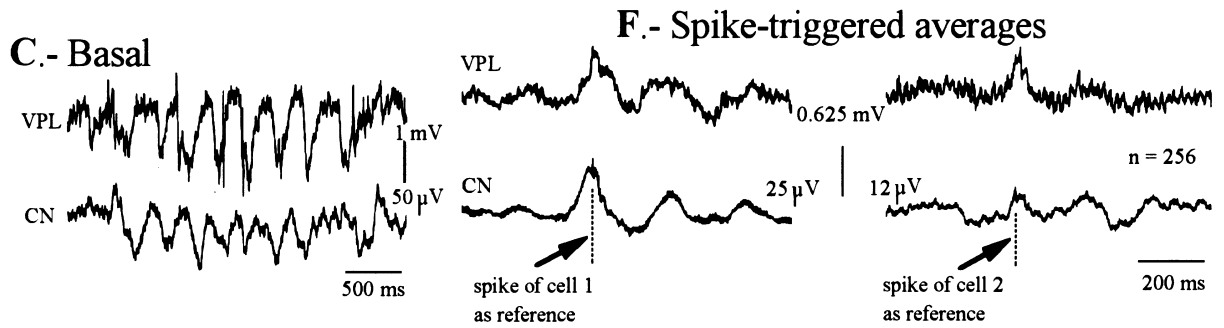

Fig. 2. Coherent delta oscillations in cuneothalamic and thalamocortical neurons. (A) VPL-thalamocortical neuron responding antidromically to internal capsula (IC) and monosynaptically to medial lemniscus (ML) stimulation. (B) ML stimulation permitted to identify a cuneothalamic (cell 2) and an interneuron (cell 1) within the middle main cuneate nucleus. (C) The oscillating synaptic noise of the thalamocortical cell was tightly coupled to the extracellular field potentials recorded in the cuneate in the frequency range of delta rhythms. (D) The auto-correlations show the similarity of rhythmic activity among the three cells. (E) Cross-correlations using the cuneate cells as reference demonstrate the resonant activity showed by the three cells. (F) Using cuneate cell 1 (left) and cuneate cell 2 (right) as reference to compile spike-triggered averages of all three cells (256 trigger events; the averages of the cuneate neurons respect to themselves) demonstrated coherent oscillations within the delta range. Stimulus artefacts marked by black dots (ML) and by an arrowhead (IC).

lami could be mimicked in the cuneate by the shell of the GABAergic interneurons surrounding the cuneothalamic cells. Since the cuneate interneurons are monosynaptically activated by descending cortical input and inhibit the cuneothalamic cells [3], the cortical outputs to the thalamus and to the cuneate, although mostly using different corticofugal cells, could impose a similar oscillating slow rhythmicity on both structures.

In contrast to spindles, the delta waves can be generated in single thalamic neurons by the interplay of two intrinsic membrane conductances, the hyperpolarization-activated cation current $\left(I_{\mathrm{h}}\right)$ and the transient low-threshold calcium current $\left(I_{t}\right)$ [17]. When extracellular recordings of cuneothalamic cells were analyzed unfiltered, field potentials within the delta range were discovered. Fig. 2A shows the identification of a VPL-thalamocortical cell responding antidromically to IC stimulation (A, right) and monosynaptically to increasing intensities of ML stimulation (A, left). Two cuneate cells were simultaneously recorded, one (cell 2 in Fig. 2B) was a cuneothalamic neuron, and the other did not follow 100/s iterative ML stimulation (Fig. $2 \mathrm{~B}$, right) and was therefore considered as an interneuron. Coherent oscillating activity within the delta frequency was detected between cuneothalamic and thalamocortical cells (Fig. 1E and 2C). Autocorrelations (Fig. 2D), crosscorrelations (Fig. 2E) and spike-triggered averages using the spikes of the cells 1 or 2 of Fig. 2B as triggers (Fig. 2F), demonstrated the time-coincidence between both cuneate extracellular single responses and thalamic intracellular spikes, as well as the time-locking between the delta oscillating rhythms. This appears to corroborate previous data indicating that the cuneate cells posses intrinsic membrane rhythmicities similar to those described in thalamic neurons [3]. Thus, in the chloralose anesthetized cat, the cuneate and VPL thalamic cells show strongly coupled slow rhythms as well as coherent oscillations within the delta frequency. As stated previously, the slow rhythms could be imposed by the cerebral cortex. The delta cuneate rhythmicity might be an intrinsically generated phenomenon similarly to what occurs in thalamic neurons. The coupling of delta rhythms between cuneate and thalamic activities might represent a general state of quiet sleep in which the cuneate cells could coherently oscillate together with other structures such as the thalamo-cortico-thalamic networks. In addition, preliminary data obtained in our laboratory indicate that stimulation of the peripheral receptive fields replaces the oscillating mode of the cuneate cells into a tonic (transfer) mode, like it occurs in thalamic neurons. 
Therefore, the cuneate prethalamic cells would have a more similar behavior with the thalamic cells than previously thought. Thus, cuneate cells might be able to rhythmically encode their outputs.

This work was supported by a grant from the DGICYT (PB93-0345). J. Mariño is a postgraduate fellow of the Xunta de Galicia.

[1] Bentivoglio, M. and Rustioni, A., Corticospinal neurons with branching axons to the dorsal column nuclei in the monkey, J. Comp. Neurol., 253 (1986) 260-276.

[2] Berrevoets, C.E. and Kuypers, H.G.J.M., Pericruciate cortical neurons projecting to brainstem reticular formation, dorsal column nuclei and spinal cord in the cat, Neurosci. Lett., 1 (1975) 257262.

[3] Canedo, A., Primary motor cortex influences on descending and ascending systems, Prog. Neurobiol., (1996) in press.

[4] Canedo, A. and Lamas, J.A., Pyramidal and corticospinal synaptic effects over reticulospinal neurones in the cat, J. Physiol., 463 (1993) 475-489.

[5] Deschênes, M., Paradis, M., Roy, J.P. and Steriade, M., Electrophysiology of neurons of lateral thalamic nuclei in cat: resting properties and burst discharges, J. Neurophysiol., 51 (1984) 1196-1219.

[6] Fyffe, R.E.W., Cheema, S.S. and Rustioni, A., Intracellular staining study of the feline cuneate nucleus. I. Terminal patterns of primary afferent fibers, J. Neurophysiol., 56 (1986) 1268-1283.

[7] Kuypers, H.G.J.M. and Tuerk, J.D., The distribution of the cortical fibers within the nuclei cuneatus and gracilis in the cat, J. Anat., 98 (1964) 143-162.

[8] Jahnsen, H., Electrophysiological characteristics of neurons in the guinea pig deep cerebellar nuclei in vitro, J. Physiol., 372 (1986) 129-147.

[9] Lamas, J.A., Martinez, L. and Canedo, A., Pericruciate fibres to the red nucleus and to the medial bulbar reticular formation, Neuroscience, 62 (1994) 115-124.

[10] Martinez, L., Lamas, J.A. and Canedo, A., Pyramidal tract and corticospinal neurons with branching axons to the dorsal column nuclei of the cat, Neuroscience, 68 (1995) 195-206.

[11] Pinault, P. and Deschênes, M., The origin of rhythmic fast subthreshold depolarizations in thalamic relay cells under urethane anaesthesia, Brain Res., 595 (1992) 295-300.

[12] Rustioni, A. and Hayes, N.L., Corticospinal tract collaterals to the dorsal column nuclei of cats, Exp. Brain Res., 43 (1981) 237-245.

[13] Steriade, M. and Llinás, R., The functional states of the thalamus and the associated neuronal interplay, Physiol. Rev., 68 (1988) 649-742.

[14] Steriade, M., Núñez, A. and Amzica, F., A novel slow $(<1 \mathrm{~Hz})$ oscillation of neocortical neurons in vivo: depolarizing and hyperpolarizing components, J. Neurosci., 13 (1993) 3252-3265.

[15] Steriade, M., Núñez, A. and Amzica, F., Intracellular analysis of relations between the slow $(<1 \mathrm{~Hz})$ neocortical oscillation and other sleep rhythms of the electroencephalogram, J. Neurosci., 13 (1993) 3266-3283.

[16] Steriade, M., Contreras, D., Curró Dossi, R. and Núñez, A., The slow $(<1 \mathrm{~Hz})$ oscillation in reticular thalamic and thalamocortical neurons: scenario of sleep rhythm generation in interacting thalamic and neocortical networks, J. Neurosci., 13 (1993) 3284-3299.

[17] Steriade, M., McCormick, D.A. and Sejnowski, T.J., Thalamocortical oscillations in the sleeping and aroused brain, Science, 262 (1993) 679-685.

[18] Steriade, M., Contreras, D. and Amzica, F., Synchronized sleep oscillations and their paroxysmal developments, Trends Neurosci., 17 (1994) 199-208. 